\title{
Article
}

\section{PPARGC1A gene polymorphism is associated with exercise-induced fat loss}

Mazur, luliia lu, Drozdovska, Svitlana, Andrieieva, Olena, Vinnichuk, Yulia, Polishchuk, Anna, Dosenko, Victor, Andreev, Igor, Pickering, Craig and Ahmetov, Ildus I.

Available at http://clok.uclan.ac.uk/34827/

Mazur, Iuliia lu, Drozdovska, Svitlana, Andrieieva, Olena, Vinnichuk, Yulia, Polishchuk, Anna, Dosenko, Victor, Andreev, Igor, Pickering, Craig and Ahmetov, Ildus I. (2020) PPARGC1A gene polymorphism is associated with exercise-induced fat loss. Molecular Biology Reports . ISSN 0301-4851

It is advisable to refer to the publisher's version if you intend to cite from the work. http://dx.doi.org/10.1007/s11033-020-05801-z

For more information about UCLan's research in this area go to http://www.uclan.ac.uk/researchgroups/ and search for < name of research Group>.

For information about Research generally at UCLan please go to http://www.uclan.ac.uk/research/

All outputs in CLoK are protected by Intellectual Property Rights law, including Copyright law. Copyright, IPR and Moral Rights for the works on this site are retained by the individual authors and/or other copyright owners. Terms and conditions for use of this material are defined in the policies page.

\section{CLoK}

Central Lancashire online Knowledge www.clok.uclan.ac.uk

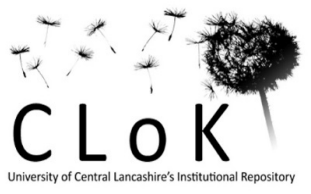


PPARGC1A gene polymorphism is associated with exercise-induced fat loss

Iuliia Iu. MAZUR ${ }^{1 *}$, Svitlana DROZDOVSKA ${ }^{1}$, Olena ANDRIEIEVA ${ }^{1}$, Yulia VINNICHUK ${ }^{1}$, Anna POLISHCHUK ${ }^{1}$, Victor DOSENKO ${ }^{2}$, Igor ANDREEV ${ }^{3}$, Craig PICKERING ${ }^{4}$, Ildus I. AHMETOV 5,6,7

${ }^{1}$ National University of Physical Education and Sport of Ukraine, Kyiv, Ukraine

${ }^{2}$ Bogomoletz Institute of Physiology, National Academy of Sciences of Ukraine, Kyiv, Ukraine

${ }^{3}$ Institute of Molecular Biology and Genetics, National Academy of Sciences of Ukraine, Kyiv, Ukraine

${ }^{4}$ Institute of Coaching and Performance, School of Sport and Wellbeing, University of Central Lancashire, Preston, UK

${ }^{5}$ Laboratory of Molecular Genetics, Kazan State Medical University, Kazan, Russia

${ }^{6}$ Department of Physical Education, Plekhanov Russian University of Economics, Moscow, Russia

${ }^{7}$ Research Institute for Sport and Exercise Sciences, Liverpool John Moores University, Liverpool, UK

*Corresponding author: Iuliia Iu. Mazur, Medical and Biology department, National University of Physical Education and Sport of Ukraine, 1 Physkultury st., 03150, Kyiv, Ukraine. E-mail: Yuliya.vorona@gmail.com ORCHID ID 0000-0002-2151-1825 


\begin{abstract}
BACKGROUND:

Obesity is a widespread problem within modern society, serving to increase the risk of cardiovascular, metabolic, and neurodegenerative disorders. Peroxisome proliferator-activated receptor gamma (PPAR $\gamma$ ) and PPAR $\gamma$ coactivator $1 \alpha(\mathrm{PGC} 1 \alpha)$ play a key role in the regulation of cellular energy metabolism and is implicated in the pathology of these diseases. This study examined the association between polymorphisms of the PPARG and PPARGC1A genes and individual variability in weight loss in response to physical activity intervention.

\section{METHODS AND RESULTS}

Thirty-nine obese Ukrainian women $\left(44.4 \pm 7.5\right.$ years, BMI > $\left.30.0 \mathrm{~kg} / \mathrm{m}^{2}\right)$ undertook a 3-month fitness program whilst following a hypocaloric diet ( 1500 calories). Anthropometric and biochemical measurements took place before and after the program. Single nucleotide polymorphisms within or near $P P A R G(\mathrm{n}=94)$ and PPARGC1A $(\mathrm{n}=138)$ were identified and expression of PPARG mRNA was measured via reverse transcription and amplification. The association between DNA polymorphisms and exercise-induced weight loss, initial body mass, biochemistry and PPARG expression was determined using one-way analysis of variance (ANOVA).

The present intervention induced significant fat loss in all participants (total fat: $40.3 \pm 5.3$ vs $36.4 \pm 5.7 \%$; $P<0.00001$ ). Only one polymorphism (rs17650401 C/T) within the PPARGC1A gene was found to be associated with fat loss efficiency after correction for multiple testing, with $\mathrm{T}$ allele carriers showing the greatest reduction in body fat percentage (2.5-fold; $P=0.00013)$ compared to non-carriers.
\end{abstract}

\title{
CONCLUSIONS:
}

PPARGC1A (rs17650401) is associated with fat loss efficiency of the fitness program in obese women. Further studies are warranted to test whether this variation is associated with fat oxidation.

Key words:

PPARG, obesity, peroxisome proliferator-activated receptor, Weight loss intervention, Single-nucleotide polymorphism

\section{Introduction}

Peroxisome proliferator-activated receptors (PPARs) are a group of nuclear receptors demonstrated to play a key role in carbohydrate and lipid metabolism, from the single cell to the whole organism. These receptors are classified into three families; PPAR $\alpha, \operatorname{PPAR} \delta$, and PPAR $\gamma$ PPAR $\gamma$ controls lipid storage and synthesis[1]. Its activation leads to triacylglycerol production and accumulation in liver, differentiation and proliferation of adipocytes, increased synthesis of both adiponectin and resistin [2], and regulation of the immune system response via a reduction in inflammatory activity of lymphocytes [3, 4]. Peroxisome proliferator-activated receptor-gamma coactivator (PPARGC1A) is a transcriptional coactivator that regulates the genes involved in energy production [5]. Additionally, PPARGC1A is involved in NO-induced mitochondrial biogenesis, along with switching of muscle metabolism towards oxidative phosphorylation, thus adapting muscle tissue to endurance training [6]. A number of single nucleotide polymorphisms (SNPs) within the PPAR gene family are 
associated with a predisposition to metabolic, cardiovascular, and neurodegenerative disorders, such as obesity, dyslipidemia, hypertension, cancer, type 2 diabetes, and Parkinson's disease[7], [8].

Obesity is a major problem within modern society, with the percentage of affected adults in comprising of up to $40 \%$ of the population in many developed countries[9]. Obesity is associated with an increased prevalence of cardiovascular diseases, type 2 diabetes, gastrointestinal and oncological disorders[10], [11]. Designing a personalized weight loss strategy may be an effective tool in reducing the spread of mentioned diseases [12].

To date, there is a limited data demonstrating the effects of SNPs within the genes of the PPAR family on weight loss through increased physical activity. PPARG polymorphisms are known to influence variability of body mass index (BMI) independently of sex, age, energy intake, and physical activity[13]. However, the effects of these polymorphisms on exercise-induced weight loss have not been previously characterized. The purpose of this study, therefore, was to identify SNPs within PPARG and PPARGC1A associated with the efficacy of moderate physical exercise on a weight loss in population of obese women.

\section{Materials and methods}

\section{Participants}

The study participants were 39 Ukrainian middle-aged women (44.4 \pm 5.8 years of age), of which $32 \%$ had no previous physical activity experience. The inclusion criteria were: a BMI of greater than 30, and an absence of biochemical signs of severe metabolic syndrome (i.e. normal LDL, HDL, cholesterol and triglycerides). General characteristics of the study participants are shown in the Table 1.

\section{Intervention}

All participants were enrolled into a 3-month fitness program, which included 180 minutes of aerobic exercise per week with an average metabolic equivalent (MET) value of 6. Depending on the baseline physical fitness levels of each participant, exercise heart rate did not exceed 115-125 bpm (low fitness), 125-135 bpm (moderate fitness), or 135-145 bpm (well-adapted). Heart rate was measured using Polar RC3 GPS with Polar heart rate sensor H7 (Finland). Additionally, throughout the study period, the participants were advised to maintain a healthy hypocaloric diet ( 1500 calories), avoid high-sugar, high-fat, and processed food diets, and to include more vegetables and wholemeal products in their diet.

\section{Anthropometric and Blood Measurements}

All blood and anthropometric measurements were done before and after the exercise lifestyle intervention. BMI was calculated as body weight in kilograms divided by the square of the height in meters. Percentage of total and visceral fat was measured by bioelectrical impedance analysis with "TANITA - BC-418MA" body composition analyser (Japan). For the blood biochemical test, blood samples were collected from the antecubital vein after an overnight fast. HDL, LDL, and triglycerides were measured via biochemical analyser «HYMALYZER 3000».

\section{Genotyping}

The samples of 39 subjects were sent to the commercial laboratory (Akesogen, UK), where DNA was extracted from the saliva samples using Qiagen chemistry on an automated Kingfisher FLEX instrument (Thermo Fisher Scientific, Waltham, MA, US), following the manufacturer's recommended protocols and standard operating procedures. PicoGreen and Nanodrop measurements were taken to measure the quality and quantity of the DNA. Input to the custom testing array occurs at $200 \mathrm{ng}$ in $20 \mu \mathrm{l}$. Amplification, fragmentation, and 
resuspension was performed using Biomek FXP following Affymetrix's high throughput protocol for Axiom 2.0. Hybridization was performed for 24 hours at $48{ }^{\circ} \mathrm{C}$ in a Binder oven, and staining and scanning of the arrays (94 PPARG and 138 PPARGC1A SNPs; DNAFit's custom microchips) were performed using GeneTitan instrumentation (Thermo Fisher Scientific, Waltham, MA, US), all following the same Affymetrix high throughput Axiom 2.0 protocol. Data analysis was then performed using a raw CEL file data input into the Affymetrix Axiom Analysis Suite (Affymetrix, Santa Clara, CA, US).

\section{Analysis of mRNA expression of PPARG}

RNA was obtained from blood plasma. The analysis of mRNA expression of PPARG was performed only at the start of the weight loss program. Expression of $P P A R G$ mRNA was measured via reverse transcription and amplification. Reverse transcription was performed with Random Hexamer primer, Revert Aid RT, Ribo Lock RNAse inhibitor and dNTP mixture on Gene Amp ${ }^{\circledR}$ PCR System 2700, Applied Biosystems, USA. The samples were incubated at $42^{\circ} \mathrm{C}$ for $1 \mathrm{~h}$, followed by heating at $70^{\circ} \mathrm{C}$ for $10 \mathrm{~min}$. Amplification was performed on a 7500 FastReal-time PCR system (Applied Biosystems, USA). The values were corrected for $\beta$-actin mRNA expression.

\section{Statistical analysis}

Statistical analysis was conducted using RStudio software. One-way analysis of variance (ANOVA) was applied to determine statistical significance among different groups. The paired t-test was used to detect the significance of dynamic changes. $P<0.05$ was considered statistically significant. Bonferroni's correction for multiple testing was performed by dividing the $P$ value by the number of tests where appropriate.

\section{Results}

\section{Effects of intervention program}

The changes in body composition and biochemical profile in response to the 3-month exercise training and hypocaloric diet are summarized in Table 1 . As expected, the intervention induced significant improvements of triglycerides, LDL, and cholesterol levels, as well as BMI, waist circumference, hip circumference, total and visceral fat.

\section{Influence of SNPs on PPARG mRNA expression}

The mean value of PPARG mRNA levels corrected for $\beta$-actin mRNA expression was $38.3 \pm 1.73$. Of the 94 analysed PPARG SNPs, the only one SNP rs6442311 A/G within PPARG intron was associated with PPARG mRNA expression after correction for multiple testing. The rs6442311 AG genotype was associated with increased $P P A R G$ expression $(9.01 \pm 0.07 \% ; P=0.022)$.

\section{Associations between PPARG and PPARGC1A SNPs and adipose tissue mass}

We evaluated relationships between 94 PPARG and 138 PPARGC1A SNPs and baseline values of body fat percentage, visceral fat, and markers of lipid metabolism (HDL, LDL, cholesterol). Of the tested PPARG SNPs, only one (rs6442311) of the 94 showed a significant $(P=0.016)$ influence on the initial percentage of body adipose tissue. For individuals with the AG genotype of rs6442311 the mean values of body fat percentage and visceral fat were $29.3 \%$ and $5.5 \%$ respectively. For individuals with the AA genotype, the results were $41.6 \%$ and $10.5 \%$, respectively (Fig. 1). Accordingly, the minor allele of rs6442311 had a negative correlation with the accumulation of white adipose tissue, especially of visceral fat, and with a higher 
ratio of total to visceral fat mass (5.33 for carriers and 3.96 for non-carriers). All genotypes frequencies are summarized in Table II.

Of the 136 tested PPARGC1A SNPs, only rs6846769 showed a significant association with baseline percentage of adipose tissue $(P=0.034)$. More specifically, the minor allele was correlated with a lower body fat percentage; $41.4 \%$ and $28 \%$ for major and minor alleles, respectively (Fig.1). Furthermore, the percentage of visceral fat in minor allele carriers was almost two-times lower (9.7\% and $5 \%$ ), and the ratio of total adipose fat to visceral fat was higher in carriers (5.6) than in non-carriers (4.3).

\section{Associations between PPARGC1A SNPs and blood lipoproteins}

None of the 94 tested PPARG SNPs showed a statistically significant association with HDL, LDL, cholesterol or triglycerides. One of the 136 tested PPARGC1A SNPs showed a significant influence on blood lipoproteins. The minor allele of rs4458444 (intron variant) was associated with increased HDL $(P=0.0081)$, and total cholesterol $(P=0.036)$. One of the 136 tested PPARGC1A SNPs demonstrated correlation with increased triglyceride blood concentrations. A mean triglyceride concentration of $2.38 \mathrm{mM} / 1$ was found in carriers of rs2305681 minor allele, whereas the mean of carriers of major allele was $1.6 \mathrm{mM} / 1$.

\section{Associations between PPARG and PPARGC1A SNPs and fat mass loss}

Only one polymorphism (rs17650401 C/T) within the PPARGC1A gene was found to be associated with fat loss efficiency after correction for multiple testing, with $\mathrm{T}$ allele carriers showing the greatest exercise-induced reduction in body fat percentage $(2.5$-fold; $P=0.00013)$ compared to non-carriers. As for the 94 analyzed PPARG SNPs, rs9833097 $(P=0.00023)$ and rs12629751 $(P=0.0065)$ showed association with fat loss. Genotypes of rs9833097 (AA, AG, GG) were found to be associated with different degrees of weight loss $(14.60 \%, 4.71 \%$, and $2.82 \%$, respectively). Presence of the minor allele of rs12629751 was found to correlate with a twofold increase in weight loss after the three-month moderate exercise program: the percentages of body fat loss were $3.02 \%$ and $6.48 \%$ for CC and TC genotypes, respectively.

\section{Discussion}

The effectiveness of physical exercise and diet on fat loss varies considerably between individuals [13]. Our genetic association study was designed to test whether multiple variations in the PPARG and PPARGCIA genes can modulate changes in body composition and metabolic variables following a 3 months supervised fitness program and hypocaloric diet in obese Ukrainian women. We also tested the hypothesis that polymorphisms within PPARG gene may influence its expression.

PPARG plays an important role in the control of adipose tissue metabolism, adipocyte division and differentiation, fat storage and oxidation in beige and brown adipose tissue [14]. Exercise is known to increase PPARG expression and activity within skeletal muscle, thus promoting mitochondrial biogenesis and aerobic respiration [15]. An increased expression of PPARG offers protection against elevated blood glucose levels[14]; as such, the rs6442311 G allele of the PPARG gene, which has been shown in our study to be associated with increased PPARG mRNA levels, may decrease the risk of obesity-induced diabetes and cardiovascular diseases. Indeed, we found that PPARG rs6442311 G allele was also associated with a decreased baseline percentage of visceral fat.

Body fat and visceral fat percentage, and markers of lipid metabolism (HDL, LDL, cholesterol) are strongly related to the risk of metabolic syndrome, type 2 diabetes, and cardiovascular diseases [17]. Besides PPARG rs6442311, the rs6846769 polymorphism in the PPARGC1A gene showed a significant association 
with baseline percentage of adipose tissue. The rs6846769 SNP may protect against the accumulation of visceral fat in an obese person under the influence of environmental factors via the activation of adaptive thermogenesis in brown adipose tissue[15]. This SNP is located in the first intron of PPARGCIA, prior to the protein coding sequence, and may participate in the control of PPARGCIA gene expression. Enhanced PPARGC1A expression protects from visceral adipose tissue accumulation, increases total adipose to visceral fat ratio, and reduces total body fat content[16]. Furthermore, the minor allele of the PPARGC1A gene rs4458444 polymorphism was associated with increased HDL and total cholesterol. Previously[17, 18] it was shown that decreased expression of PPARGCIA may upregulate liver lipoprotein synthesis and downregulate systemic utilization of cholesterol lipoproteins. In addition, the PPARGC1A rs2305681 SNP was found to be associated with triglyceride concentration. Decreased activity of PPARGC1A is associated with increased blood triglyceride levels[7] and risk of cardiovascular disease, especially under the influence of environmental factors which induce obesity. Previously, rs2305681 showed strong correlation with anxiety disorders[22] via possible activation of the antioxidant system within GABA neurons[19]. Interestingly, PPARGC1A rs3774909 (which is in $100 \%$ linkage disequilibrium (LD) with rs2305681) has been shown previously to be associated with endurance performance[20], presumably via regulation of oxidative phosphorylation, skeletal muscle mitochondrial biogenesis, and alteration of muscle fiber types.

We have also shown that rs $17650401 \mathrm{C} / \mathrm{T}$ within the PPARGC1A gene was associated with fat loss efficiency after correction for multiple testing. This is in line with the studies showing that various types of exercises (acute, high intensity interval, and aerobic) induce PPARGC1A expression in the group of overweight people[21], which was accompanied with fatty acid oxidation[22]. Moreover, the increased PPARGC1A activity was independent of the glycemic index of diet[23] and mostly induced by the exercise program.

Furthermore, we found two candidate markers within PPARG (rs12629751 and rs9833097) that have shown association with fat loss. Interestingly, the minor allele of rs12629751, which correlated with a twofold increase in weight loss, previously demonstrated a protective property against breast cancer[24]. In addition, the polymorphism within rs7626560 (which is in LD with rs9833097) correlated with response to diabetes prevention lifestyle interventions[25], where participants were advised to perform $150 \mathrm{~min}$ of physical activity per week (in our study, participants exercised 180 min per week).

All significant SNPs identified within this research are located in the intron region. The genotype frequencies of these SNPs are listed in Table II. There is no data about the intronic SNPs effects on $P P A R G C 1 A$ or PPARG expression. Hence, these SNPs may belong to regulatory post-transcriptional sequences producing miRNA. Also, a changing of the nucleotides upstream of promotor region may lead to another nucleosome positioning, thus altering promoter strength and its interaction with transcriptional factors. Altogether, we suggest this leads to a different expression response to the same environmental factors, such as diet, age and physical exercises; this hypothesis is illustrated on Fig 2. According to our model, intronic SNPs could change positioning of nucleosomes in cis-regulatory elements, which leads to the different sensitivity of $P P A R G$ promotor region to the transcriptional factors (C/ERB, STAT5A, CREB) that are affected by hypocaloric diet or physical activity[26, 27] and other factors like polyunsaturated fatty acids, prostaglandins, and eicosanoids. Thus, physical activity might have a different impact on PPARG expression and further adipose tissue metabolism depending on a particular SNP in the intronic region. 


\section{Conclusions}

An intervention comprised of physical activity and a hypocaloric diet has a significant impact on fat loss. Although no association was found between SNPs of PPARG gene and weight loss efficiency, individual variability in fat loss efficiency may partly depend on the rs17650401 C/T polymorphism within the PPARGCIA gene. The identified SNPs, along with many others, may be helpful in designing individual weight loss programs focused on exercise and/or nutrition. According to our results, we suggest that rs17650401 CT or TT genotype carriers can effectively improve BMI through moderate aerobic exercise and hypocaloric diet, while CC genotype carriers can obtain more benefits in weight loss from additional strength exercise or highintensity interval training and increasing of omega- 3 polyunsaturated fatty acids dietary intake. These factors could have higher influence on PPARG gene and adipose tissue metabolism. Further studies are warranted to test whether this variation is associated with fat oxidation and with fat loss efficiency in other ethnicities.

\section{REFERENCES}

1. Dreyer C, Krey G, Keller H, Givel F, Helftenbein G, Wahli W (1992) Control of the peroxisomal $\beta$ oxidation pathway by a novel family of nuclear hormone receptors. Cell. 68:879-887. DOI: 10.1016/0092-8674(92)90031-7.

2. Hong F, Xu P, Zhai Y (2018) The opportunities and challenges of peroxisome proliferator-activated receptors ligands in clinical drug discovery and development. Int J Mol Sci. 19:2189. 10.3390/ijms19082189.

3. Laganà AS, Vitale SG, Nigro A (2016) Pleiotropic actions of peroxisome proliferator-activated receptors (PPARs) in dysregulated metabolic homeostasis, inflammation and cancer: Current evidence and future perspectives. Int J Mol Sci. 17:999. 10.3390/ijms17070999.

4. Korbecki J, Bobiński R, Dutka M (2019) Self-regulation of the inflammatory response by peroxisome proliferator-activated receptors. Inflamm Res. 68:443-458. 10.1007/s00011-019-01231-1.

5. Villena JA (2015) New insights into PGC-1 coactivators: Redefining their role in the regulation of mitochondrial function and beyond. FEBS J. 282:647-672. 10.1111/febs.13175.

6. Gureev AP, Shaforostova EA, Popov VN (2019) Regulation of mitochondrial biogenesis as a way for active longevity: Interaction between the Nrf2 and PGC-1 $\alpha$ signaling pathways. Front Genet. 10:1-12. 0.3389/fgene.2019.00435.

7. Diop S, Bisharat-Kernizan J, Birse R, Oldham S, Ocorr K, Bodmer R (2015) PGC-1/Spargel counteracts High-fat-diet-induced obesity and cardiac lipotoxicity downstream of TOR and brummer ATGL lipase. Cell Rep. 10:1572-1584. 10.1016/j.celrep.2015.02.022.

8. Monsalve FA, Pyarasani RD, Delgado-Lopez F, Moore-Carrasco R (2013) Peroxisome proliferatoractivated receptor targets for the treatment of metabolic diseases. Mediators Inflamm. 2013:1-18. $10.1155 / 2013 / 549627$.

9. Bray GA, Heisel WE, Afshin A (2018). The science of obesity management: An endocrine society scientific statement. Endocr Rev. 39:79-132. 10.1210/er.2017-00253.

10. Youssef J, Badr M (2011) Peroxisome proliferator-activated receptors and cancer: Challenges and opportunities. Br J Pharmacol. 164:68-82. 10.1111/j.1476-5381.2011.01383.x. 
11. Grygiel-Górniak B (2014) Peroxisome proliferator-activated receptors and their ligands: Nutritional and clinical implications - A review. Nutr J. 13:1-10. 10.1186/1475-2891-13-17.

12. Drozdovska S., Andrieieva O., Yarmak O., Blagii O. (2020) Personalization of health-promoting fitness programs for young women based on genetic factors. J Phys Edu Sport. 20:331-337. 10.7752/jpes.2020.s1046

13. Goni L, García-Granero M, Milagro FI, Cuervo M, Martínez JA (2018) Phenotype and genotype predictors of BMI variability among European adults. Nutr Diabetes. 8:1-8. 10.1038/s41387-0180041-1.

14. Shamsi BH, Ma C, Naqvi S, Xiao Y (2014) Effects of pioglitazone mediated activation of PPAR- $\gamma$ on CIDEC and obesity related changes in mice. PLoS One. 9:1-8. 10.1371/journal.pone.0106992

15. Puigserver P, Wu Z, Park CW, Graves R, Wright M, Spiegelman BM (1998) A cold-inducible coactivator of nuclear receptors linked to adaptive thermogenesis. Cell. 92:829-839. 10.1016/s0092$8674(00) 81410-5$

16. Singh SP, Schragenheim J, Cao J, Falck JR, Abraham NG, Bellner L (2016) PGC-1 alpha regulates HO-1 expression, mitochondrial dynamics and biogenesis: Role of epoxyeicosatrienoic acid. Prostaglandins Other Lipid Mediat. 125:8-18. 10.1016/j.prostaglandins.2016.07.004

17. Estall JL, Kahn M, Cooper MP (2009) Sensitivity of lipid metabolism and insulin signaling to genetic alterations in hepatic peroxisome proliferator-activated receptor- $\gamma$ coactivator- $1 \alpha$ expression. Diabetes. 58:1499-1508. 10.2337/db08-1571

18. Liu Y, Zhai J, Chen J, Wang X, Wen T (2019) PGC-1 $\alpha$ protects against oxidized low-density lipoprotein and luteinizing hormone-induced granulosa cells injury through ROS-p38 pathway. Hum Cell. 32:285-296. 10.1007/s13577-019-00252-6

19. St-Pierre J, Drori S, Uldry M (2006) Suppression of reactive oxygen species and neurodegeneration by the PGC-1 transcriptional coactivators. Cell. 127:397-408. 10.1016/j.cell.2006.09.024

20. Sarzynski MA, Rankinen T, Sternfeld B (2013) Association of single-nucleotide polymorphisms from 17 candidate genes with baseline symptom-limited exercise test duration and decrease in duration over 20 years: The coronary artery risk development in young adults (CARDIA) fitness study. Circ Cardiovasc Genet. 3:531-538. 10.1161/CIRCGENETICS.110.957183

21. Vargas-ortiz K, Victoriano P, Mac MH (2019) Exercise and sirtuins : A way to mitochondrial health in skeletal muscle. In J Mol Sci. 20:1-11. 10.3390/ijms20112717

22. Zheng W, Rogoschin J, Niehoff A (2018) Combinatory effects of phytoestrogens and exercise on body fat mass and lipid metabolism in ovariectomized female rats. J Steroid Biochem Mol Biol. 178:73-81. 10.1016/j.jsbmb.2017.11.002

23. Mulya A, Haus JM, Solomon TPJ (2017) Exercise training-induced improvement in skeletal muscle PGC-1 $\alpha$-mediated fat metabolism is independent of dietary glycemic index. Obesity. 25:721-729. 10.1002/oby.21799

24. Lee E, Hsu C, Van Den Berg D (2012) Genetic variation in peroxisome proliferator-activated receptor gamma, soy, and mammographic density in Singapore Chinese women. Cancer Epidemiol Biomarkers Prev. 21:635-644. 10.1158/1055-9965.EPI-11-1042 
25. Jablonski KA, McAteer JB, De Bakker PIW (2010) Common variants in 40 genes assessed for diabetes incidence and response to metformin and lifestyle intervention in the diabetes prevention program. Diabetes. 59:2672-2681. 10.2337/db10-0543.

26. Shen H., Tong L., Balazs R., Cotman CW (2001) Physical activity elicits sustained activation of the cyclic AMP response element-binding protein and mitogen-activated protein kinase in the rat hippocampus. Neuroscience. 107(2):219-29. 10.1016/s0306-4522(01)00315-3.

27. Consitt LC, Wideman L, Hickey M.S., Morrison R.F (2008). Phosphorylation of the JAK2-STAT5 pathway in response to acute aerobic exercise. Med Sci Sports Exerc. 40(6): 1031-1038. 10.1249/MSS.0b013e3181690760

\section{Declarations}

Funding. There were no additional souses of funding.

Conflicts of interest. CP is a former employee of DNAfit Life Sciences, a genetic testing company. The remaining authors declare no conflicts of interest. The funding sponsors had no role in the design of the study; in the collection, analyses, or interpretation of data; in the writing of the manuscript; or in the decision to publish the results.

Ethics approval. This study was approved by the National Academy of Science of Ukraine Bogomoletz Institute of Physiology Biomedical Ethics Committee, Kyiv, Ukraine (\#1/18 05.04.2018). Written informed consent was obtained from the participants, and the study conformed to the guidelines and principles of the Declaration of Helsinki.

Consent to publication. All authors transfer the ownership of copyright to the journal Molecular Biology Reports should their work be published in this journal.

Authors' contributions. IIM contributed to data collection, data analysis, interpretation of results and manuscript writing; SD participated in the design of the study, data analysis, interpretation of results and manuscript writing;

OA, YV, AP and IA contributed to data collection, data analysis and interpretation of results; VD participated in the design of the study, data analysis and interpretation of results; CP participated in the interpretation of results and manuscript writing; IIA participated in the design of the study, contributed to data analysis, interpretation of results and manuscript writing. All authors have read and approved the final version of the manuscript, and agree with the order of presentation of the authors.

TABLES

Table I.-Effects of intervention program in 39 obese women

\begin{tabular}{|l|l|l|l|}
\hline \multirow{2}{*}{ Trait } & \multicolumn{2}{c|}{ Changes (mean \pm SD) } & \multirow{2}{*}{ P } \\
\cline { 2 - 3 } & \multicolumn{1}{|c|}{ before } & \multicolumn{1}{c|}{ after } & \\
\hline Triglycerides, mM/l & $1.58 \pm 0.78$ & $1.47 \pm 1.04$ & 0.756 \\
\hline LDL, mM/l & $3.21 \pm 1.16$ & $2.86 \pm 0.98$ & $0.016^{*}$ \\
\hline HDL, mM/l & $1.44 \pm 0.3$ & $1.41 \pm 0.28$ & 0.549 \\
\hline Cholesterol, mM/l & $5.32 \pm 1.23$ & $4.81 \pm 1.08$ & $0.022^{*}$ \\
\hline BMI, kg/m ${ }^{2}$ & $33.2 \pm 2.9$ & $30.4 \pm 3.7$ & $<0.00001^{*}$ \\
\hline
\end{tabular}




\begin{tabular}{|l|l|l|l|}
\hline Waist circumference, $\mathrm{cm}$ & $92.4 \pm 8.9$ & $85.9 \pm 8.9$ & $<0.00001^{*}$ \\
\hline Hip circumference, $\mathrm{cm}$ & $115.3 \pm 7.1$ & $107.9 \pm 7.7$ & $<0.00001^{*}$ \\
\hline Total fat, \% & $40.3 \pm 5.3$ & $36.4 \pm 5.7$ & $<0.00001^{*}$ \\
\hline Visceral fat, \% & $9.5 \pm 2.4$ & $8.1 \pm 2.2$ & $<0.00001^{*}$ \\
\hline
\end{tabular}

Table II. - Genetic frequencies of identified SNP in population of 39 obese women

\begin{tabular}{|l|c|l|l|l|}
\hline \multicolumn{1}{|c|}{ SNPs } & $\begin{array}{c}\text { Frequencies of a } \\
\text { minor allele }\end{array}$ & \multicolumn{3}{|c|}{ Genotypes frequencies } \\
\hline rs6442311 & 0.026 & AA 0.947 & AG 0.053 & GG 0.000 \\
\hline rs6846769 & 0.013 & AA 0.974 & AC 0.026 & CC 0.000 \\
\hline rs4458444 & 0.013 & GG 0.974 & GA 0.026 & AA 0.000 \\
\hline rs17650401 & 0.064 & CC 0.872 & CT 0.128 & TT 0.000 \\
\hline rs9833097 & 0.192 & GG 0.667 & GA 0.308 & AA 0.026 \\
\hline rs12629751 & 0.115 & CC 0.769 & CT 0.231 & TT 0.000 \\
\hline rs3774909 & 0.051 & CC 0.923 & CT 0.051 & TT 0.026 \\
\hline
\end{tabular}

\section{TITLES OF FIGURES}

Figure 1.- Associations between PPARG rs6442311 and PPARGClA rs6846769 polymorphisms and percentage of total and visceral adipose tissue in obese women $\left(\mathrm{BMI}>30 \mathrm{~kg} / \mathrm{m}^{2}\right)$.

Figure 2.- Theoretical model of intron SNPs influence on PPARG expression under moderate-exercise environment. 